\title{
El plan estratégico de marketing como mecanismo de posicionamiento. caso dirección de vinculación ESPOCH
}

\begin{abstract}
(c) (1) (9)(2)
The strategic marketing plan as a positioning mechanism. case management of linking ESPOCH
\end{abstract}

Yolanda Patricia Moncayo Sánchez. ${ }^{1}$, Marco Vinicio Salazar Tenelanda. ${ }^{2}$ \& Edison Ruperto Carrillo Parra. ${ }^{3}$

Recibido: 07-05-2020 /Aceptado: 06-06-2020/ Publicado: 03-07-2020

DOI: https://doi.org/10.33262/concienciadigital.v3i3.1295

\begin{abstract}
.
The research was carried out in order to design marketing strategies to position the ESPOCH Liaison Office in the city of Riobamba, Chimborazo Province, 2019, therefore, the following objectives were set: General Objective to design a strategic marketing plan for the positioning of the ESPOCH Liaison Directorate in the city of Riobamba, Chimborazo Province, year 2017. Specific Objectives to establish the corporate philosophy of the ESPOCH Liaison Directorate. Prepare the situational diagnosis based on an internal and external analysis. Design marketing strategies in order to improve the structural performance and the positioning of the Management, to efficiently face the institutional challenges. The methodology used was based on the application of surveys applied to students, political teachers and the business sector of the city of Riobamba, in addition to the preparation of the diagnosis that knew the current situation of the Directorate. It is determined according to the results that the design and implementation of strategies that help improve the internal structure of the same is necessary, since the low positioning, limited communication, and low presence in social networks has mainly had the ignorance of the Management mentioned above and its main axes of action. The proposal is based on developing strategies that help position the Directorate in the city of Riobamba. In this way it will try to solve the main problems that the institution has and specifically the
\end{abstract}

\footnotetext{
${ }^{1}$ Escuela Superior Politécnica de Chimborazo, FADE, Riobamba, Ecuador, pattymoncayo_s@yahoo.es

${ }^{2}$ Escuela Superior Politécnica de Chimborazo, FADE, Riobamba, Ecuador, msalazar_t@espoch.edu.ec

${ }^{3}$ Escuela Superior Politécnica de Chimborazo, FADE, Riobamba, Ecuador, victor.avalos@espoch.edu.ec
} 
aforementioned department, and its effective component goes hand in hand with the degree of positioning that it achieves with this plan based on specific strategies.

Keywords: Strategies, positioning, Corporate Image.

\section{Resumen.}

La investigación se realizó con el fin de diseñar estrategias de marketing para posicionar la Dirección de Vinculación de la ESPOCH en la ciudad de Riobamba, Provincia de Chimborazo, año 2019, por lo tanto, se plantearon los siguientes objetivos: Objetivo General Diseñar un plan estratégico de marketing para el posicionamiento de la Dirección de Vinculación de la ESPOCH en la ciudad de Riobamba, Provincia de Chimborazo, año 2017. Objetivos Específicos Establecer la filosofía corporativa de la Dirección de Vinculación de la ESPOCH. Elaborar el diagnóstico situacional basado en un análisis interno y externo. Diseñar estrategias de marketing a fin de mejorar el desempeño estructural y el posicionamiento de la Dirección, para enfrentar eficientemente los desafíos institucionales. La metodología utilizada se basó en la aplicación de encuestas aplicadas a los estudiantes, docentes politécnicos y al sector empresarial de la ciudad de Riobamba, además de la elaboración del diagnóstico que permitió conocer la situación actual de la Dirección. Se determinó según los resultados obtenidos que son necesarios el diseño e implementación de estrategias que ayuden a mejorar la estructura interna de la misma, puesto que el bajo posicionamiento, limitada comunicación, y la baja presencia en redes sociales principalmente ha causado el desconocimiento de la Dirección antes mencionada y sus principales ejes de acción. La propuesta se fundamentó en elaborar estrategias que ayuden a posicionar a la Dirección en la ciudad de Riobamba. De esta manera se tratará de resolver los principales problemas que la institución posee y específicamente el departamento antes mencionado, y su componente efectivo va de la mano con el grado de posicionamiento que este alcance con este plan basado en estrategias específicas.

Palabras clave: Estrategias, posicionamiento, Imagen Corporativa.

\section{Introducción.}

El Según el autor Sainz De Vicuña (2012), al hablar del plan estratégico de la organización, nos estamos refiriendo al plan maestro en el que la alta dirección recoge las decisiones estratégicas corporativas que ha adaptado "hoy" en referencia a lo que hará en los tres próximos años (horizonte más habitual del plan estratégico), para lograr una organización más competitiva que le permita satisfacer las expectativas de sus diferentes grupos de intereses. Por lo tanto, el estudio desarrolló una investigación sobre el plan estratégico de marketing el cual permita posicionar la Dirección de Vinculación de la ESPOCH como el un departamento de seguimiento a graduados, gestión y formación intercultural y servicio a la comunidad en la ciudad de Riobamba.

En la investigación presentada a continuación se procura reflejar las diferentes formas a tomar en consideración en el posicionamiento, así como las estrategias adecuadas para ser implementadas por la Dirección, de forma que permita y estimule mantener un buen posicionamiento en el mercado de manera permanente y eficaz. 
El interés de realizar esta investigación, surge a pesar de que la Dirección ya lleva un buen tiempo constituida en la institución, y al ver que no ha alcanzado el auge de posicionamiento esperado, a diferencia del departamento de Vinculación de la UNACH que ha tenido una gran aceptación por parte del sector empresarial y de la ciudadanía Riobambeña

Se identificó la problemática, así como los objetivos de investigación tanto general como específicos, al mismo tiempo que se convirtieron en la base sobre la cual se fundamenta el presente trabajo de investigación.

El marco teórico es integrar el tema de la investigación con las teorías, enfoques teóricos, estudios y antecedentes en general que se refieren al problema de investigación. En tal sentido el marco teórico según Tamayo (2012) nos amplía la descripción del problema. Integra la teoría con la investigación y sus relaciones mutuas.

Por lo cual la investigación incluye un marco teórico el cual fundamenta desde el punto de vista teórico varias definiciones que abarca temas como planeación estratégica, componentes de la planeación, análisis de la situación, interno y externo, posicionamiento, entre otros.

El marco metodológico contiene el método de investigación, como el cualitativo, también se determina la población y muestra que sirvieron de base para la obtención de información mediante encuestas.

Según Laura Fischer y Jorge Espejo, la estrategia de mercadotecnia "comprende la selección y el análisis del mercado, es decir, la elección y el estudio del grupo de personas a las que se desea llegar, así como la creación y permanencia de la mezcla de mercadotecnia que las satisfaga"

En ese sentido, la propuesta de la investigación, contiene el diseño de estrategias de marketing a fin de mejorar el desempeño estructural y el posicionamiento de la Dirección, para enfrentar eficientemente los desafíos institucionales, en el mercado objeto de estudio.

\section{El problema.}

La Dirección de Vinculación pertenece a un eje principal de la Escuela Superior Provincia de Chimborazo ubicada en la ciudad de Riobamba en la panamericana sur Km 1 1/2, provincia de Chimborazo, cuenta con 3 dependencias dentro de la Dirección las mismas que son: seguimiento a graduados, servicio a la comunidad, formación y gestión intercultural. Cabe indicar que viene funcionando a partir del año 2014, por lo que al ser una Dirección eminentemente nueva o recién creada carece de una estructura organizativa, de identidad y de informes o estudios estratégicos que conlleven a una gestión eficiente y generadora de valor tanto para el mercado interno como externo, a fin de alcanzar las metas y objetivos planteados. 
Según información histórica de la Dirección de Vinculación, se observa la inexistencia de documentos o informes técnicos o de planeación a largo plazo; puesto que las decisiones se toman sobre la marcha y responden en su mayoría a actividades específicas.

La tendencia a la obtención de resultados inmediatos, la dificultad para la previsión de eventos futuros, la ausencia de una estructura organizativa y el bajo nivel de conocimiento tanto del público interno como externo incide en el posicionamiento y en la eficiencia administrativa de la Unidad de Vinculación de la ESPOCH.

Por lo anterior mencionado se evidencia la ausencia de un documento técnico que oriente la toma de decisiones acertadas a largo, como es la planeación o plan estratégico

\section{Marco teórico conceptual.}

\section{Fundamentación teórica.}

\section{Marketing.}

Por su parte (Stanton, Michael, \& Walker, 2007, pág. 9), explican que el concepto de marketing "hace hincapié en la orientación del cliente y en la coordinación de las actividades de marketing para alcanzar los objetivos de desempeño de la organización".

En ese sentido, y a criterio de los autores, "el concepto de marketing se basa en tres ideas:

- Toda la planeación y las operaciones deben orientarse al cliente. Esto es, cada departamento y empleado deben aplicarse a la satisfacción de las necesidades de los clientes.

- Todas las actividades de marketing de una organización deben coordinarse. Esto significa que los esfuerzos de marketing (planeación de producto, asignación de precios, distribución y promoción) deben idearse y combinarse de manera coherente, congruente, y que un ejecutivo debe tener la autoridad y responsabilidad totales del conjunto completo de actividades de marketing.

- El marketing coordinado, orientado al cliente, es esencial para lograr los objetivos de desempeño de la organización. El desempeño de un negocio es generalmente medido en términos de recuperación de la inversión, precio de almacén y capitalización de mercado. Sin embargo, el objetivo inmediato podría ser algo menos ambicioso que mueva a la organización más cerca de su meta definitiva".

\section{Planeación estratégica.}


Para (Agueda \& Olarte, pág. 35) la planeación estratégica es el arte y la ciencia de formular, implementar y evaluar las decisiones internacionales que permiten a la organización alcanzar sus objetivos".

Además, indica que la planificación estratégica es un proceso de evaluación sistemática de la naturaleza de un negocio, definiendo los objetivos a largo plazo, identificando metas y objetivos cuantitativos, desarrollando estrategias para alcanzar dichos objetivos y localizando recursos para llevar a cabo dichas estrategias.

Siendo así una poderosa herramienta de diagnóstico, análisis, reflexión y toma de decisiones colectivas, en torno al quehacer actual y al camino que deben recorrer en el futuro las organizaciones e instituciones, para adecuarse a los cambios y a las demandas que les impone el entorno y lograr el máximo de eficiencia y calidad de sus prestaciones.

Por otro lado (Rugman, Alan, 2006, pág. 76) afirma que la planeación estratégica observa la cadena de consecuencias de causas y efectos en la organización durante un tiempo, relacionada con una decisión real o intencionada que tomará el director. La esencia de la planeación estratégica consiste en la identificación sistemática de los puntos fuertes, débiles y limitaciones, tanto propias como del entorno; en especial deberán evaluarse las fuerzas de los competidores y sus planes sobre futuras acciones, así como una serie de oportunidades y amenazas que se derivan de la situación de la propia empresa en relación con el entorno en el que se actúa.

\section{Los componentes de la planeación estratégica.}

Para (Lopez, Maria Elvira, 2013, págs. 9-10) los componentes son esenciales en la planeación estratégica debido a que con ellos se sabe claramente lo que se va hacer o proponer y son los siguientes:

Misión: enunciado que refleja el objetivo fundamental de la empresa.

Valores: conjunto de enunciados que reflejan los principios fundamentales bajo los cuales debe operar la empresa.

Estrategia: patrón o plan que integra las principales metas y políticas de una organización, y a la vez establece la secuencia coherente de las acciones a realizar.

Metas u objetivos: establecen qué es lo que se va a lograr y cuándo serán alcanzados los resultados, pero no establecen cómo serán logrados.

Políticas: son reglas o guías que expresan los límites dentro de los que debe ocurrir la acción.

Programas: especifican la secuencia de las acciones necesarias para alcanzar los 
Decisiones estratégicas: son aquéllas que establecen la orientación general de una empresa y su viabilidad máxima a la luz, tanto de los cambios predecibles como de

Los impredecibles que, en su momento, puedan ocurrir en los ámbitos que son de su

Interés o competencia

\section{Características de la planeación estratégica.}

(Lerma, Alejandro \& Bácena Sergio, 2012, pág. 17) Afirman que cada proceso de planeación varía de una organización a otra, existen ciertos trazos generales que pueden percibirse en toda planeación estratégica, siendo las características las siguientes:

- Objetivos viables, cuantificables y conocidos por todos.

- Coherencia homogeneidad en las decisiones

- Medición mediante la eficiencia

\section{Importancia de la planificación estratégica.}

(Rodríguez, J., 2005, pág. 45) Señala que la importancia de la planificación estratégica radica en que es un proceso de evaluación sistemática de la naturaleza de un negocio, en el cual se definen los objetivos a largo plazo, se identifican metas y objetivos cuantitativos, se desarrollan estrategias para alcanzar dichos objetivos y se localizan recursos para llevar a cabo dichas estrategias.

Además (Arranz, Antonio, 2010, pág. 65) indica que la planificación estratégica se convierte en una poderosa herramienta de diagnóstico, análisis, reflexión y toma de decisiones colectivas, en torno al quehacer actual y al camino que deben recorrer en el futuro las organizaciones e instituciones, para adecuarse a los cambios y a las demandas que les impone el entorno y lograr el máximo de eficiencia y calidad en los productos y servicios que ofrecen.

\section{Beneficios de la planificación estratégica.}

(Steiner, G., 2007, pág. 56) Afirma que la planificación estratégica permite que la organización tome parte activa, en lugar de reactiva, en la configuración de su futuro, es decir, la organización puede emprender actividades e influir en ellas (en lugar de sólo responder) y, por consiguiente, puede controlar su destino.

Por otro lado (Castellano, Luis, 2015, pág. 44) señala que los beneficios de la planificación estratégica son vitales para una empresa u organización, citando los siguientes:

- Permite detectar oportunidades, y clasificarlas en prioridad y explotarlas

- Ofrece una visión objetiva de los problemas administrativos

- Representa un marco para coordinar y controlar mejor las actividades

- Reduce consecuencias de condiciones y cambios adversos 
- Permite tomar decisiones importantes para respaldar mejor los objetivos establecidos

- Permite asignar con mayor eficacia recursos y tiempo a oportunidades detectadas

- Fomenta el razonamiento anticipándose a los hechos

- Propicia actitud positiva ante el cambio

\section{Limitaciones de la planeación estratégica.}

Según (Mintzberg, H. \& Quinn, J., 1998) existen tres falsedades de la planificación estratégica:

Falacia de la predeterminación: la planificación estratégica no sólo requiere un seguimiento de los pronósticos, sino también estabilidad durante la creación de la estrategia.

Falacia de separación: si el sistema se ocupa de pensar, entonces el pensamiento debe estar separado de la acción, la estrategia de las operaciones (tácticas), la formulación de la aplicación, los que piensan de los que hacen y los estrategas de los objetos de sus estrategias

Falacia de formalización: las investigaciones indican que la creación de estrategias es un proceso tremendamente complejo que comprende los procesos más sofisticados, sutiles y a veces subconscientes del conocimiento humano y social.

Inevitablemente, los estrategas efectivos exhiben ciertas cualidades sobresalientes y, aunque actúan en forma premeditada, suelen dejar de lado los planes formales para aparecer como visionarios informales.

\section{Proceso de planeación estratégica.}

(Lambin, Jean, 2005, pág. 90) Indica que el proceso de planeación estratégica tiene, básicamente cuatro componentes: la misión, los objetivos, las estrategias y el plan de cartera. El desarrollo del proceso da como resultado un plan estratégico. Que son:

a. Definir la misión de la organización: Toda organización tiene una misión que la define el tener bien claro la misión hace que se tenga muy claro el espacio del producto (manufactura, servicio o idea).

b. Establecer los objetivos de la organización. Los objetivos son el fundamento de cualquier programa de planeación. La misión aclara el propósito de la organización a la administración.

c. Formular las estrategias de la organización: El propósito de las estrategias es determinar y comunicar. 


\section{Plan estratégico de marketing.}

En términos generales, el plan estratégico de marketing es un documento escrito que incluye una estructura compuesta por: 1) un análisis de la situación, 2) los objetivos de marketing, 3) el posicionamiento y la ventaja diferencial, 4) la descripción de los mercados meta hacia los que se dirigirán los programas de marketing, 5) el diseño de la mezcla de marketing y 6) los instrumentos que permitirán la evaluación y control constante de cada operación planificada, (Lambin, Jean, 2005, pág. 569).

\section{El contenido del Plan Estratégico de Marketing}

Para (Lambin, Jean, 2005, pág. 67) El plan estratégico es un documento escrito que incluye una estructura de seis puntos muy importantes (los cuales son adaptados a las necesidades de cada empresa u organización):

\section{Análisis de la situación.}

Identificación de la empresa: Descripción de objetivos, misión, visión, reseña histórica, servicios que oferta, objetivo social.

Análisis de factores externos e internos Son aquellos que afectan directamente a los resultados de la institución.

\section{Análisis interno.}

Según (Lerma, Alejandro \& Bácena Sergio, 2012, pág. 151) el análisis interno consiste en estudiar a fondo rubros y actividades que son controlables por la organización. Es útil para hacer comparaciones con la competencia, pues un análisis interno puede arrojar insuficiencias o ventajas relativas de la UEN.

El análisis interno comprende la relación y descripción de las fuerzas y debilidades propias.

Fuerzas: Todo tipo de atributos, capacidades, destrezas, recursos que colocan a la persona o empresa en una situación de ventaja respecto de la competencia.

Debilidades: Pueden encontrarse como limitaciones internas que se convierten en desventajas al contrastar ciertas áreas de la empresa con respecto de otras empresas. Dificultan la consecución de metas y objetivos.

\section{Análisis AMOFITH.}

Para realizar este análisis se tiene como herramientas el análisis de las áreas funcionales que integran el ciclo operativo de la organización. Este análisis interno permitirá mostrar la actual situación de la empresa/ organización basado en el análisis de siete áreas funcionales, las cuales son: administración y gerencia, marketing y ventas, operaciones y 
logística, finanzas y contabilidad, recursos humanos, sistemas de información y tecnología. (Mendoza, Pedro, 2000, pág. 89)

\section{Análisis externo.}

(Fred, David, 2008, págs. 82-83) Afirma que el objetivo del análisis externo es desarrollar una lista finita de oportunidades que podrían beneficiar a una empresa y de amenazas que ésta debería evitar. El análisis externo no se enfoca en desarrollar una lista exhaustiva de todos los posibles factores que pudieran influir en la empresa; más bien, pretende identificar las variables clave que ofrecen respuestas factibles. Las empresas deben ser capaces de responder tanto ofensiva como defensivamente a los factores mediante la formulación de estrategias que aprovechen las oportunidades externas o reduzcan al mínimo el efecto de las posibles amenazas.

\section{Análisis PEST.}

(Castellano, Luis, 2015, pág. 89) Indica que el análisis PEST es una herramienta de gran utilidad para comprender el crecimiento o declive de un mercado, y en consecuencia, la posición, potencial y dirección potencial de un negocio. Es una herramienta de medición de negocios. PESTE está compuesta por las iniciales de factores Políticos, Económicos, Sociales y Tecnológicos, utilizados para evaluar el mercado en el que se encuentra un negocio o unidad.

El PEST funciona como un marco para analizar la situación, y como el análisis DOFA, el cual está basado en factores internos (fortalezas y debilidades) y externos (oportunidades y amenazas).

\section{Análisis DOFA.}

(Serna, Humberto, 1994, pág. 140) Señala que es un acrónimo de debilidades, oportunidades, fortalezas y amenazas. El análisis DOFA ayuda a determinar si la organización está capacitada para desempeñarse en su medio. Mientras más competitiva en comparación con sus competidores esté la empresa mayores probabilidades tiene de éxito. Esta simple noción de competencia conlleva consecuencia poderosa para el desarrollo de una estrategia efectiva. El análisis DOFA, así como el de vulnerabilidad integran el diagnóstico estratégico y lo hace por tanto Global. 
Tabla 1. Ejemplo análisis FODA

\begin{tabular}{lcc}
\hline & Fortalezas & Debilidades \\
\hline Análisis & Capacidades distintas & Recursos y capacidades escasas \\
Interno & Ventajas naturales & Resistencia al cambio \\
& Recursos superiores & Problemas de motivación del personal \\
& Oportunidades & Amenazas \\
\hline Análisis & Nuevas tecnologías & \\
Externo & Debilitamiento de & \\
& competidores & \\
& Posicionamiento estratégico & \\
& Altos riesgos - Cambios en el & \\
& entorno & \\
\hline
\end{tabular}

Fuente: (Serna, Humberto, 1994, pág. 140)

\section{Estudio de mercado.}

Según ( Hatton, A., 2012, pág. 68) dice que durante mucho tiempo se ha pensado que dentro del análisis de la situación, este era el único punto que debía desarrollarse para confeccionar un plan de marketing y siempre a través de una encuesta; paradójicamente no siempre es necesario hacerla para conocer tanto el mercado como la situación de la que se parte, ya que en la actualidad existen, como hemos explicado en el capítulo de investigación de mercados, alternativas muy válidas para obtener información fiable.

\section{Determinación de objetivos.}

Según (Kotler, P. y Armstrong, G, 2012, pág. 56) señala que los objetivos constituyen un punto central en la elaboración del plan de marketing, ya que todo lo que les precede conduce al establecimiento de los mismos y todo lo que les sigue conduce al logro de ellos. Los objetivos en principio determinan numéricamente dónde queremos llegar y de qué forma; estos además deben ser acordes al plan estratégico general, un objetivo representa también la solución deseada de un problema de mercado o la explotación de una oportunidad.

\section{Características de los objetivos.}

(Serna, Humberto, 1994, pág. 45) Indica que en el establecimiento de objetivos lo que más se persigue es la fijación del volumen de ventas o la participación del mercado con el menor riesgo posible, para ello los objetivos deben ser:

- Viables. Es decir, que se puedan alcanzar y que estén formulados desde una óptica práctica y realista.

- Concretos y precisos. Totalmente coherentes con las directrices de la compañía.

- En el tiempo. Ajustados a un plan de trabajo. 
- Consensuados. Englobados a la política general de la empresa, han de ser aceptados y compartidos por el resto de los departamentos.

- Flexibles. Totalmente adaptados a la necesidad del momento.

- Motivadores. Al igual que sucede con los equipos de venta, estos deben constituirse con un reto alcanzable.

\section{Elaboración y selección de estrategias.}

(Quintal, A., 2005, pág. 89) indica que las estrategias son los caminos de acción de que dispone la empresa para alcanzar los objetivos previstos; cuando se elabora un plan de marketing estas deberán quedar bien definidas de cara a posicionarse ventajosamente en el mercado y frente a la competencia, para alcanzar la mayor rentabilidad a los recursos comerciales asignados por la compañía.

Asimismo, se debe ser consciente de que toda estrategia debe ser formulada sobre la base del inventario que se realice de los puntos fuertes y débiles, oportunidades y amenazas que existan en el mercado, así como de los factores internos y externos que intervienen y siempre de acuerdo con las directrices corporativas de la empresa.

\section{Plan de acción.}

Por lo otro lado (Stanton, Michael, \& Walker, Fundamentos de Marketing, 2007, pág. 67) manifiestan que, si se desea ser consecuente con las estrategias seleccionadas, tendrá que elaborarse un plan de acción para conseguir los objetivos propuestos en el plazo determinado. Cualquier objetivo se puede alcanzar desde la aplicación de distintos supuestos estratégicos y cada uno de ellos exige la aplicación de una serie de tácticas. Estas tácticas definen las acciones concretas que se deben poner en práctica para poder conseguir los efectos de la estrategia. Ello implica necesariamente el disponer de los recursos humanos, técnicos y económicos, capaces de llevar a buen término el plan de marketing.

\section{Establecimiento de presupuesto.}

Finalmente (Agueda \& Olarte, 2008, pág. 89) indican que una vez que se sabe qué es lo que hay que hacer, solo faltan los medios necesarios para llevar a cabo las acciones definidas previamente. Esto se materializa en un presupuesto, cuya secuencia de gasto se hace según los programas de trabajo y tiempo aplicados. Para que la dirección general apruebe el plan de marketing, deseará saber la cuantificación del esfuerzo expresado en términos monetarios, por ser el dinero un denominador común de diversos recursos, así como lo que lleva a producir en términos de beneficios, ya que a la vista de la cuenta de explotación provisional podrá emitir un juicio sobre la viabilidad del plan o demostrar interés de llevarlo adelante. Después de su aprobación, un presupuesto es una autorización para utilizar los recursos económicos. No es el medio para alcanzar un objetivo, ese medio es el programa.

\section{Posicionamiento.}


Para (Kotler, Philip \& Armstrong, Gary, 2012, pág. 207) El posicionamiento además de decidir a qué segmentos de mercado se va a dirigir, la empresa debe determinar una proposición de valor, es decir de qué manera creará un valor diferenciado para los segmentos meta, y que posiciones desea obtener en tales segmentos. La posición de un producto es la forma en que los consumidores definen el producto con base en sus atributos importantes; es decir, el lugar que ocupa en la mente de los consumidores, en relación con los productos de la competencia.

(Ries, Al \& Trout, Jack, 1992, pág. 3) Indica que el posicionamiento comienza con un producto, que puede ser un artículo, un servicio, una compañía, una institución o incluso una persona. Quizá usted mismo.

Pero el posicionamiento no se refiere al producto, sino a lo que se hace con la mente de los probables clientes; o sea, cómo se ubica el producto en la mente de éstos.

Por lo mismo es errado llamar a este concepto "posicionamiento no comporte un cambio. Sí lo comporta. Pero los cambios que se hacen a los nombres, a los precios y al embalaje no son en realidad cambios del producto mismo.

El posicionamiento es también lo primero que viene a la mente cuando se trata de resolver el problema de cómo lograr ser escuchado en una sociedad súper comunicada.

\section{Objetivo del posicionamiento.}

El posicionar correctamente un servicio en el mercado consiste en hacerlo más deseable, compatible y aceptable y relevante para el segmento meta, diferenciándolo del ofrecido por la competencia, es decir ofrecer un servicio que sea efectivamente percibido como "único" por los clientes. Un servicio, al estar bien posicionado, hace que el segmento lo identifique perfectamente como una serie de deseos y necesidades de su propia escala de valores, haciendo que el grado de lealtad de sí mismo sea mayor y más fuerte respecto a los ofrecidos por los competidores" [...] (Del Valle Quevedo, 2008).

\section{Formas de posicionamiento.}

Existe varias formas para desarrollar una estrategia de posicionamiento, como ejemplo podemos citar algunas:

- Por atributos: La crema CREST lucha contra la caries.

- Por precio, calidad: SAGA FALA- BELLA es una tienda que "vale".

- Por aplicación: ALIVIUM para el dolor.

- Por usuario del producto: CRISTAL para el pueblo, consumidores de cerveza.

- Por clase de producto: MILO: alimento para niños. 
La selección de uno o más mercados meta por la gerencia de mercadotecnia, establece la etapa de desarrollo para cada mercado meta.

Un mercado meta es cualquier grupo de clientes potenciales hacia quienes una compañía decide dirigir su oferta de mercadotecnia.

El posicionamiento se determina por las percepciones de los compradores sobre la marca de la empresa en relación con las marcas de sus competidores directos; por ello, lo esencial es cómo es percibido el programa de mercadotecnia de la empresa (producto, precio, distribución y comunicación) por las personas que están en el segmento meta, en relación con los programas de mercadotecnia de los competidores.

\section{Selección de una estrategia de diferenciación y posicionamiento.}

A algunas compañías les resulta fácil elegir su estrategia de diferenciación y posicionamiento. Por ejemplo, una empresa reconocida por su calidad en ciertos segmentos buscará esta posición en un nuevo segmento, si existen nuevos suficientes compradores que busquen calidad. Sin embargo, en muchos casos, dos o más empresas buscarán la misma posición, por lo que cada una tendrá que encontrar otras formas de distinguirse. Cada empresa debe diferenciar su oferta creando un conjunto único de beneficios que atraiga a un grupo sustancial dentro del segmento. (Kotler, Philip \& Armstrong, Gary, 2012, pág. 208)

\section{Las bases de un posicionamiento.}

(Win (1982, págs. 79-88) ha identificado seis tipos de posicionamientos posibles para una marca. Son las siguientes:

- Un posicionamiento basado en una cualidad distintiva del producto.

- Un posicionamiento basado en las ventajas o en la solución aportada.

- Un posicionamiento basado en una oportunidad de utilización especifica.

- Un posicionamiento orientado a una categoría de usuarios.

- Un posicionamiento en relación a una marca competidora.

- Un posicionamiento de ruptura en relación a la categoría del producto.

Se puede igualmente adoptar un posicionamiento que se apoya en un estilo de vida específico. (Lambin, Jean, 2005, págs. 219-220)

\section{Metodología.}


La modalidad de la investigación se sustenta bajo un enfoque mixto: cualitativocuantitativo. Cualitativo porque tiene una concepción lineal, es decir que hay claridad entre los elementos que conforman el problema; mientras que la modalidad cuantitativa es aquella que permite examinar los datos de manera numérica, especialmente en el campo de la Estadística, según el autor (Calero, JL., 2000, pág. 59).

\section{Población y Muestra.}

Al conjunto de unidades de análisis que concuerdan con una serie de especificaciones basadas en los objetivos de estudio, se le conoce con el nombre de población, o sea, la población se refiere a la totalidad de los elementos que poseen las principales características objeto de análisis. (Cortés,2012, pág.61, citado en Guadalupe, V. 2017).

En la investigación realizada se aplicó la fórmula de cálculo para un universo finito de población, considerando que la misma contempla las unidades de muestreo objeto de análisis.

Los datos referidos al cálculo de la población y muestra son tomados de, Guadalupe, V. (2017). Diseño de un Plan Estratégico de Marketing para posicionar la Dirección de Vinculación de la Espoch, en la ciudad de Riobamba, Provincia De Chimborazo (Tesis de Pregrado). Escuela Superior Politécnica de Chimborazo, Riobamba, Ecuador.

Se ha seleccionado dos segmentos para aplicar las encuestas, siendo el primero estudiantes y docentes de la ESPOCH y el segundo segmento las empresas de la ciudad de Riobamba.

\section{Segmento uno.}

Cálculo de la muestra a estudiantes y docentes de la ESPOCH

Por lo mencionado anteriormente y de acuerdo a datos de la secretaria general de la ESPOCH el total de estudiantes Politécnicos matriculados para el periodo marzo-agosto 2018 es de 14.534 estudiantes, por otro lado, el total de docentes titulares y ocasionales de las siete facultades según fuentes de la página del LOTAIP/ ESPOCH / MAYO del mismo año es de 1.150 , dando un total del universo de 15.684

\section{Segmento dos.}

Sector empresarial de la ciudad de Riobamba.

Se ha tomado como referencia 51 empresas que constan en la nómina del catastro municipal de Riobamba 2018, convirtiéndose de esta manera en una población finita/ alcanzable y accesible por lo que se encuestó a toda la población, la mayoría de ellas situadas en el parque industrial de la ciudad.

\section{Resultados.}




\section{Encuesta 1 (estudiantes y docentes):}

Tabla 2. Conocimiento la Dirección de Vinculación

\begin{tabular}{ccc}
\hline Descripción & Frecuencia & Porcentaje \\
\hline $\mathrm{Si}$ & 255 & $68 \%$ \\
No & 120 & $32 \%$ \\
Total & $\mathbf{3 7 5}$ & $\mathbf{1 0 0 \%}$ \\
\hline
\end{tabular}

Fuente: Elaboración propia.

Tabla 3. Conocimiento sobre los 3 ejes principales de la Dirección

\begin{tabular}{ccc}
\hline Descripción & Frecuencia & Porcentaje \\
\hline Servicio a la comunidad & 91 & $27 \%$ \\
Seguimiento a graduados & 66 & $19 \%$ \\
Formación y gestión intercultural & 184 & $54 \%$ \\
Total & $\mathbf{3 4 1}$ & $\mathbf{1 0 0 \%}$ \\
\hline
\end{tabular}

Fuente: Elaboración propia.

Tabla 4. Planificación estratégica

\begin{tabular}{ccc}
\hline Descripción & Frecuencia & Porcentaje \\
\hline $\mathrm{Si}$ & 13 & $3 \%$ \\
$\mathrm{No}$ & 362 & $97 \%$ \\
Total & $\mathbf{3 7 5}$ & $\mathbf{1 0 0 \%}$ \\
\hline
\end{tabular}

Fuente: Elaboración propia.

Tabla 5. Medio/os de comunicación para recibir información

\begin{tabular}{ccc}
\hline Descripción & Frecuencia & Porcentaje \\
\hline Canal tvs & 32 & $9 \%$ \\
Canal ecuavision & 23 & $6 \%$ \\
Facebook & 86 & $23 \%$ \\
Instagram & 38 & $10 \%$ \\
Twitter & 31 & $8 \%$ \\
Correo electrónico & 36 & $10 \%$ \\
Página web & 65 & $17 \%$ \\
Diario la prensa & 33 & $9 \%$ \\
Diario los andes & 21 & $6 \%$ \\
Radio local & 10 & $3 \%$ \\
Total & $\mathbf{3 7 5}$ & $\mathbf{1 0 0 \%}$ \\
\hline
\end{tabular}

Fuente: Elaboración propia. 
Tabla 6. Isla informativa

\begin{tabular}{ccc}
\hline Descripción & Frecuencia & Porcentaje \\
\hline $\mathrm{Si}$ & 271 & $72 \%$ \\
$\mathrm{No}$ & 104 & $28 \%$ \\
Total & $\mathbf{3 7 5}$ & $\mathbf{1 0 0 \%}$ \\
\hline
\end{tabular}

Fuente: Elaboración propia.

Tabla 7. Tipos de publicidad

\begin{tabular}{ccc}
\hline Descripción & Frecuencia & Porcentaje \\
\hline Revistas informativas & 37 & $9 \%$ \\
Trípticos & 51 & $11 \%$ \\
Hojas volantes & 44 & $12 \%$ \\
Vallas publicitarias & 74 & $18 \%$ \\
Rótulos luminosos & 63 & $15 \%$ \\
Pantallas electrónicas & 118 & $28 \%$ \\
Rotulación de vehículos & 28 & $7 \%$ \\
Total & $\mathbf{4 1 5}$ & $\mathbf{1 0 0 \%}$ \\
\hline
\end{tabular}

Fuente: Elaboración propia.

Tabla 8. Publicidad impresa/ ubicación

\begin{tabular}{ccc}
\hline Descripción & Frecuencia & Porcentaje \\
\hline A la entrada de la espoch & 159 & $42 \%$ \\
En un punto estratégico de cada facultad & 143 & $38 \%$ \\
Junto al bar de su escuela & 41 & $11 \%$ \\
En las afueras de la biblioteca de su & 32 & $9 \%$ \\
Total & $\mathbf{3 7 5}$ & $\mathbf{1 0 0 \%}$ \\
\hline
\end{tabular}

Fuente: Elaboración propia.

Tabla 9. Publicidad visual/ubicación

\begin{tabular}{ccc}
\hline Descripción & Frecuencia & Porcentaje \\
\hline A la entrada de la espoch & 110 & $29 \%$ \\
En los espacios recreativos de la & 148 & $39 \%$ \\
Internamente en las calles de la & 64 & $17 \%$ \\
Sectores céntricos de la ciudad & 53 & $14 \%$ \\
Total & $\mathbf{3 7 5}$ & $\mathbf{1 0 0 \%}$ \\
\hline
\end{tabular}

Fuente: Elaboración propia.

\section{Encuesta 2 (sector empresarial)}


Tabla 10. Tipo de empresa

\begin{tabular}{ccc}
\hline Descripción & Frecuencia & Porcentaje \\
\hline Empresas privadas & 39 & $76 \%$ \\
Empresas públicas & 12 & $24 \%$ \\
Total: & $\mathbf{5 1}$ & $\mathbf{1 0 0} \%$ \\
\hline
\end{tabular}

Fuente: Elaboración propia

Tabla 11. Conocimiento de la Dirección

\begin{tabular}{ccc}
\hline Descripción & Frecuencia & Porcentaje \\
\hline $\mathrm{Si}$ & 17 & $33 \%$ \\
$\mathrm{No}$ & 34 & $67 \%$ \\
Total & $\mathbf{5 1}$ & $100 \%$ \\
\hline
\end{tabular}

Fuente: Elaboración propia.

Tabla 12. Conocimiento de una Ins. Superior

\begin{tabular}{ccc}
\hline Descripción & Frecuencia & Porcentaje \\
\hline No & 26 & $51 \%$ \\
Si & 25 & $49 \%$ \\
Total: & $\mathbf{5 1}$ & $\mathbf{1 0 0} \%$ \\
\hline
\end{tabular}

Fuente: Elaboración propia.

Tabla 13. Medios de comunicación

\begin{tabular}{ccc}
\hline Descripción & Frecuencia & Porcentaje \\
\hline Televisión local & 2 & $4 \%$ \\
Redes sociales y pág. & 30 & $59 \%$ \\
Web & 3 & $6 \%$ \\
Radio local & 15 & $29 \%$ \\
Correo electrónico & 1 & $2 \%$ \\
Material impreso & $\mathbf{5 1}$ & $\mathbf{1 0 0 \%}$ \\
Total. &
\end{tabular}

Fuente: Elaboración propia

Tabla 14. Disposición asistencia a seminario

\begin{tabular}{ccc}
\hline Descripción & Frecuencia & Porcentaje \\
\hline $\mathrm{Si}$ & 41 & $80 \%$ \\
$\mathrm{No}$ & 10 & $20 \%$ \\
Total: & $\mathbf{5 1}$ & $\mathbf{1 0 0 \%}$ \\
\hline
\end{tabular}


Fuente: Elaboración propia.

Tabla 15. Asistencia técnica

\begin{tabular}{ccc}
\hline Descripción & Frecuencia & Porcentaje \\
\hline $\mathrm{Si}$ & 44 & $86 \%$ \\
$\mathrm{No}$ & 7 & $14 \%$ \\
Total: & $\mathbf{5 1}$ & $\mathbf{1 0 0 \%}$ \\
\hline
\end{tabular}

Fuente: Elaboración propia.

\section{Discusión:}

Entre los principales aspectos determinados en el segmento uno, tenemos: el 54\% de los encuestados identifica a la Dirección de Vinculación únicamente como una dependencia de gestión intercultural; el 89\% desconoce sobre la ejecución de proyectos y/o convenios de vinculación; los medios de comunicación más requeridos son: Facebook y pagina web; otro tipo de publicidad que le gustaría la población encuestada es mediante un canal de comunicación directo a través de una isla informativa; así como también anuncios en pantallas electrónicas, vallas publicitarias y rótulos luminosos.

En el segmento dos, el $67 \%$ de las empresas responden que no saben que la ESPOCH cuenta con la Dirección de Vinculación y la mayoría de ellos desconoce de una institución superior con la que puedan realizar convenios y proyectos; los medios por los que desean obtener información al respecto son: redes sociales y pagina web; el 80\% manifestó el interés por asistir a seminarios informativos; el 86\% mostró la intención de recibir asistencia técnica y otros estudios con la ESPOCH.

\section{Hallazgos de la encuesta aplicada al sector empresarial de Riobamba.}

El 67\% de las empresas encuestadas responden que no saben que la ESPOCH cuenta con la Dirección de Vinculación y la mayoría de ellos desconoce de una institución superior con la que puedan realizar convenios y proyectos, mientras que del $49 \%$ que afirma tener conocimiento al respecto el $39 \%$ de ellos mantienen algún tipo de proyecto con la UNACH.

Los medios por los que desean obtener información al respecto son: redes sociales y pagina web.

El 80\% manifiesta estar de acuerdo en asistir a seminarios informativos.

En cuanto a la intención de recibir asistencia técnica, elaboración de promedios y otros estudios con la ESPOCH, el 86\% responde que si está interesado en asistir.

Según datos de la encuesta aplicada al sector empresarial y a los estudiantes y docentes Politécnicos la mayoría de ellos desconocen de la existencia y de los ejes principales que desempeña la Dirección de Vinculación de la ESPOCH y por ello es necesario el desarrollo 
de un plan estratégico de marketing que ayude a ver los puntos fuertes y débiles de la Dirección para tomar las decisiones más adecuadas y construir posteriormente estrategias que mejoren y ayuden a posicionarse en el sector que brinda el servicio.

\section{Estrategias de Posicionamiento.}

Tomando como base los resultados de la investigación, se plantea las siguientes estrategias:

- Fortalecimiento del subsistema de servicio a la comunidad mediante la figura de asistente administrativo y marketing con el objeto de fortalecer la estructura interna de la Dirección de Vinculación y lograr la oferta de un servicio a la colectividad mejorado y eficiente.

- Implementación de publicidad exterior mediante la instalación de una pantalla eléctrica, con el objeto de dar a conocer la existencia de la Dirección de Vinculación y su razón de ser en el público de la ciudad de Riobamba.

- Implementación de islas informativas dentro y fuera de la institución, a fin de dar a conocer al segmento interno y externo las actividades y el ámbito de acción de la Dirección de Vinculación.

- Implementación de publicidad exterior mediante la colocación de vallas publicitarias, resaltando imágenes culturales propias de la Dirección y señalando claramente los contactos de interacción.

- Diseño suvenires y material impreso con el objeto de general además una publicidad utilitaria que motive a los segmentos objetivos a conservar la misma.

\section{Conclusiones:}

- La investigación de mercado permitió determinar los requerimientos del público objetivo a la Dirección, de Vinculación lo que conlleva a generar una estructuración interna eficiente y competitiva.

- El diagnóstico situacional demuestra una baja posición en la estructura interna, debido a que el subsistema servicio a la colectividad dispone de personal limitado, el mismo que cumple multifunciones y al mismo tiempo descuida las actividades que su manual determina y así también genera actividades que no le dan responsabilidad, pero las cumple por mandato superior.

- Se determinó que el público objetivo de la ciudad Riobamba desconoce el actuar de la Dirección de Vinculación de la Espoch, lo que hace prever un planteamiento estratégico inmediato, que ayude al posicionamiento como marca y de servicio de la institución. 
- La investigación demuestra una gran necesidad de tomar decisiones estratégicas con el objeto de visibilizar el accionar de la Dirección de Vinculación en beneficio de la población interna y externa con información específica que pueda ser analizada por la comunidad.

- Se determina que el éxito de cualquier institución pública o privada va de la mano con un sistema de estrategias basadas en la necesidad de medir los objetivos dentro de KPIs, y tomando medidas alternativas al encontrar elementos que obstaculizan el proceso.

\section{Referencias bibliográficas:}

Guadalupe, V. (2017). Diseño de un Plan Estratégico de Marketing para posicionar la Dirección de Vinculación de la Espoch, en la ciudad de Riobamba, Provincia De Chimborazo (Tesis de Pregrado). Escuela Superior Politécnica de Chimborazo, Riobamba, Ecuador.

Hatton, A. (2012). La guía definitiva del plan de marketing. (Tercera Edición ed.). Madrid - España: Pearson Educación, S.A.

Hatton, A. (2012). La guía definitiva del plan de marketing. (Tercera Edición ed.). Madrid - España: Pearson Educación, S.A.

Alegre, Joaquin. (2004). La gestion del conocimiento como motor de la innovacion (Primera ed.). España: Publicacions Universitat Jaume.

Arranz, Antonio. (2010). Planeación estratégica integral:instituto internacional de capacitación y estudios. México: Pearson.

Bingham, V. D. (2009). Como entrevistar. Madrid: Rialp.

Calero, J. (2000). Investigación cualitativa y cuantitativa. Cuba: Edisec Editorial.

Castellano, Luis. (2015). Estrategia y Planificación estratégica (Primera ed.). Venezuela: IE venezuela.

Castro, A. (2017). "Manual práctico de Comunicación Organizacional" (Primera ed.). Madrid - España: Editorial Verbum.

Cohen, Willian. (2005). Plan de marketing (Segunda ed.). España: Ediciones Deusto.

Ferrel, O., Hirt, G., \& McGraw, H. (2004). ntroducción a los Negocios en un Mundo Cambiante. Mexico.

Fourez, Gerard. (2006). La construccion del conocimiento cientifico. España: Narcea Ediciones.

Frances, Antonio. (2006). Estrategia y planes para empresa. Mexico: Pearson Education.

Fred, David. (2008). Conceptos de administración estratégica (Decimoprimera edición ed.). México: Pearson educación.

González, F. (2014). "Mercadotecnia estratégica: Teoría e impacto en las unidades de información". México: Universidad Nacional Autónoma de México.

Kotler, P. (2005). En Marketing de los servicios (Cuarta edicion ed., pág. 26). España: Esic Editorial. 
Kotler, P. y Armstrong, G. (2012). “Marketing”. (Decimocuarta edición. Pág ed.). México: Pearson Educación, S.A.

Kotler, P., \& Gary, A. (2003). Fundamentos de Marketing (6ta. Edición ed.). Mexico: Prentice Hall.

Kotler, Philip \& Armstrong, Gary. (2012). Marketing (Decimocuarta edición ed.). México: Pearson editorial.

Lambin, Jean. (2005). Marketing estratégico (Tercera edicion ed.). Mc Graw Hill.

Lerma, Alejandro \& Bácena Sergio. (2012). Planeación estratégica por áreas funcionales guía práctica. (Primera edicion ed.). México: Alfomega grupo editor S.A.

López, J. (2013). Métodos e hipótesis científicas. Mexico: Edisec Editorial.

López, J. R. (2004). La Gestión por Calidad total en la Empresa Moderna. México: RAMA Alfaomega.

Lopez, Maria Elvira. (2013). Planeación estratégica un pilar en la gestión empresarial (Tercera ed.). Mexico: Itson.

McCarthy, J., \& Perrault, W. (2006). Marketing Planeación Estratégica de la Teoría a la Práctica (1er. tomo ed.). Mexico: McGraw Hil.

Mendoza, Pedro. (2000). Planeamiento estretegico en gerencia. Lima, Perú: Editorial de la Universidad Nacional Mayor de San Marcos. 


\section{PARA CITAR EL ARTÍCULO INDEXADO.}

Moncayo Sánchez, Y. P., Salazar Tenelanda, M. V., \& Carrillo Parra, E. R. (2020). El plan estratégico de marketing como mecanismo de posicionamiento. caso dirección de vinculación ESPOCH. ConcienciaDigital, 3(3), 94-115.

https://doi.org/10.33262/concienciadigital.v3i3.1295

\section{\Ciencia}

El artículo que se publica es de exclusiva responsabilidad de los autores y no necesariamente reflejan el pensamiento de la Revista Conciencia Digital.

El artículo queda en propiedad de la revista y, por tanto, su publicación parcial y/o total en otro medio tiene que ser autorizado por el director de la Revista Conciencia Digital.

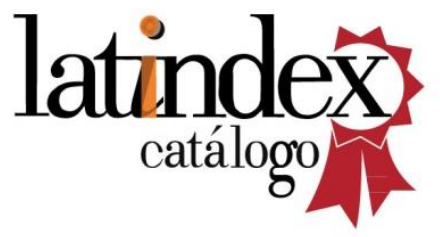

